PROCEEDINGS OF THE AMERICAN MATHEMATICAL SOCIETY

Volume 128, Number 2, Pages 325-335

S 0002-9939(99)05451-9

Article electronically published on September 27, 1999

\title{
THE PRINCIPAL AXIS THEOREM FOR HOLOMORPHIC FUNCTIONS
}

\author{
JOACHIM GRÄTER AND MARKUS KLEIN
}

(Communicated by Steven R. Bell)

\begin{abstract}
An algebraic approach to Rellich's theorem is given which states that any analytic family of matrices which is normal on the real axis can be diagonalized by an analytic family of matrices which is unitary on the real axis. We show that this result is a special version of a purely algebraic theorem on the diagonalization of matrices over fields with henselian valuations.
\end{abstract}

\section{INTRODUCTION}

This paper aims at reviewing some more or less well known material from analysis and algebra which hopefully is put into some new perspective (at least it was new for the authors) by systematically exploring the relations between algebra and analysis. It is motivated by a well known theorem of analytic perturbation theory due to Rellich [Re]: Near $z=0$, the eigenvalues of an analytic family $A(z)$ of matrices which is hermitian (resp. normal) for real values of $z$ can be chosen to be analytic, even in the case of eigenvalue degeneracies.

This theorem is presented in several textbooks (see [Ba, Ka, RS]) where it is proven by use of a rather strong algebraic result: The branching behaviour of the eigenvalues of any analytic family of matrices is explicitly given in terms of Puiseux series. The proof of Rellich's result then boils down to the observation that hermiticity (or normality) of the family excludes branching.

While Rellich's approach only leads to local solutions, methods from differential equations also provide the existence of eigenvalues, eigenprojections, and even eigenfunctions globally near the real axis provided $A(z)$ is hermitian (resp. normal) for real $z$ (cf. [Ka]).

In spite of the algebraic flavour of these results the proofs are almost purely analytic and one might ask how much analysis is really needed or how much algebra is hidden behind the analytic arguments. Since the algebraic properties of the matrices over a field $F$ are basically determined by the field $F$ itself, we have to concentrate our investigation on the algebraic properties of the field $F$ of all holomorphic functions where two cases must be distinguished: the local case, i.e. Rellich's result near a point $z=a$, and the global case near the real axis. It turns out that the global case can be reduced to the local one by elementary

Received by the editors March 6, 1998

1991 Mathematics Subject Classification. Primary 12D15, 12J10, 15A54, 34E10, 81Q15.

Key words and phrases. Valuations, Hensel's Lemma, principal axis theorem, analytic perturbation theory. 
arguments from linear algebra and the fact that $F$ can be written as $F=K(i)$ where $K$ is formally real pythagorean. From the algebraic point of view the local case is more rewarding. The ring $R$ of all holomorphic functions near a point $z=a$ is endowed canonically with a discrete valuation $v$ which counts the zeros, i.e., $v(f(z))=(v(z-a))^{n}$ if $f(z)$ has a zero of order $n$ at $z=a$. Moreover, $R$ is the corresponding valuation ring where the constants form the residue field $\bar{R} \cong \mathbb{C}$. The crucial point is that $v$ is henselian, which is an immediate consequence of the implicit function theorem for analytic functions. The connection between Hensel's Lemma and the implicit function theorem, as well as the Weierstrass preparation theorem, is one of the classical fundamental results expressing the interaction between algebra and complex analysis, resp. algebraic geometry. As soon as one realizes that $v$ is henselian, Rellich's result becomes a special version of a purely algebraic theorem on the diagonalization of matrices over fields with henselian valuations. This result is interesting in many respects. Firstly, we see that Rellich's theorem is a purely algebraic result where the implicit function theorem is the key to algebra. Secondly, Rellich's result for convergent power series also holds true for formal power series, and both cases can be treated simultaneously, since the ring of all formal power series also carries a henselian valuation with residue field $\mathbb{C}$. Finally, if one is not too enthusiastic about the algebraic approach, the algebraic arguments can be translated to complex analysis to give a new and elementary proof of Rellich's result where for instance Hensel's Lemma is replaced by the Weierstrass preparation theorem.

To make this note self-contained we proceed as follows: In Section 2, the algebraic part, we first recall the basic material from algebra up to the diagonalization of normal matrices over an appropriate class of fields. We then give a short exposition of valuation theory including two forms of Hensel's Lemma which correspond to the implicit function theorem as well as the Weierstrass preparation theorem and prove an algebraic generalization of Rellich's theorem. In Section 3, the analytic part, we explain the relevance of the algebraic results for analysis but we also sketch how a streamlined proof of the analyticity of suitable choices of eigenvalues, eigenprojections, and eigenvectors could be obtained with minimal input from algebra.

\section{The ALGEBRAic PART}

Linear algebra. In the first part of this section we provide the background from Linear Algebra, which is needed in what follows. Probably everything is more or less well known, but for the sake of completeness and readability we also keep an eye on elementary arguments.

A field $K$ is said to be formally real if -1 cannot be written as a sum of squares in $K$. While each ordered field $K$ is obviously formally real it turns out that formally real fields possess at least one ordering, i.e., as a set $K$ can be ordered linearly and for all $a, b, c \in K$ the following hold:

(i) If $a<b$, then $a+c<b+c$.

(ii) If $a<b$ and $c>0$, then $a \cdot c<b \cdot c$.

A field $K$ is said to be real closed if it is formally real, and if any algebraic extension of $K$ which is formally real must be equal to $K$. For instance, $\mathbb{C}$ is not formally real since -1 is a square in $\mathbb{C}$ and $\mathbb{R}$ is a maximal formally real subfield of $\mathbb{C}$, i.e., $\mathbb{R}$ is real closed since $\mathbb{C}$ is algebraically closed. If $K$ is formally real such 
that every sum of squares in $K$ is a square in $K$, then $K$ is called formally real pythagorean and for such a $K$ the mapping

$$
\langle\cdot, \cdot\rangle: K^{n} \times K^{n} \rightarrow K,
$$

where $\left\langle\left(u_{1}, \ldots, u_{n}\right)^{t},\left(v_{1}, \ldots, v_{n}\right)^{t}\right\rangle=u_{1} v_{1}+\ldots+u_{n} v_{n}$, is a positive definite symmetric bilinear form. Furthermore, $\langle v, v\rangle$ is the square of a uniquely determined positive element of $K$ for every nonzero $v \in K^{n}$. For instance, $\mathbb{R}$ is formally real pythagorean while $\mathbb{Q}$ is not.

Lemma 2.1. Let $K$ be a formally real pythagorean field and let $A$ be a symmetric $n \times n$ matrix over $K$ with characteristic polynomial $\chi_{A}$ such that $\chi_{A}=f \cdot g$, where $f, g \in K[x]$ are nonconstant polynomials. Then there exists an orthogonal $n \times n$ matrix $U$ over $K$ such that

$$
U^{t} A U=\left(\begin{array}{cc}
B & 0 \\
0 & B^{\prime}
\end{array}\right),
$$

where $B$, resp. $B^{\prime}$, is a symmetric $m \times m$, resp. $m^{\prime} \times m^{\prime}$, matrix over $K$ such that $1 \leq m \leq \max \{\operatorname{deg} f, \operatorname{deg} g\}$.

Proof. Let $\operatorname{deg} f \geq \operatorname{deg} g$ and put $k=\operatorname{deg} f$. If $g(A)$ is the zero matrix, then for any nonzero $v \in K^{n}$ the set $\left\{v, A v, \ldots, A^{\operatorname{deg} g-1} v\right\}$ generates a subspace $V$ of $K^{n}$ such that $1 \leq \operatorname{dim} V \leq k$ and $A w \in V$ for all $w \in V$. If $g(A) \neq 0$, then there exists $u \in K^{n}$ satisfying $v:=g(A) u \neq 0$. Since $0=\chi_{A}(A)=f(A) g(A)$ by the CayleyHamilton-Theorem we see that $\left\{v, A v, \ldots, A^{\operatorname{deg} f-1} v\right\}$ generates a subspace $V$ of $K^{n}$ having the same properties as above. Since $K$ is formally real pythagorean, we may choose an orthonormal basis $\left\{v_{1}, \ldots, v_{l}\right\}$ of $V$ and complete this set to an orthonormal basis $\left\{v_{1}, \ldots, v_{n}\right\}$ of $K^{n}$. Let $U$ be the $n \times n$ matrix over $K$ whose $i$-th column vector equals $v_{i}$. Clearly, $U$ is orthogonal and it remains to show that the $i j$-component $b_{i j}$ of $U^{t} A U$ is zero whenever $1 \leq i \leq l, l<j \leq n$. But this is obvious since $b_{i j}=\left\langle A v_{i}, v_{j}\right\rangle=0$ by $A v_{i} \in V$ and $\left\langle w, v_{j}\right\rangle=0$ for all $w \in V$ by construction of $v_{l+1}, \ldots, v_{n}$.

Corollary 2.2. Let $K$ be a formally real pythagorean field and let $A$ be a symmetric $n \times n$ matrix over $K$. Then there exists an orthogonal matrix $U$ over $K$ such that $U^{t} A U$ is diagonal if and only if the characteristic polynomial $\chi_{A}$ of $A$ splits over $K$ completely into linear factors.

If $K$ is formally real pythagorean, then $K(i)$ is a proper field extension of $K$ where $i^{2}=-1$. Every element of $K(i)$ can be written uniquely in the form $a+i b$ with $a, b \in K$ and $a-i b$ is called its conjugate. $K(i)^{n}$ is endowed canonically with a positive definite hermitian form $\langle\cdot, \cdot\rangle$, and as usual for an $n \times n$ matrix $A$ we define $A^{*}$ as the $n \times n$ matrix which can be obtained from $A^{t}$ by replacing each entry by its conjugate. Furthermore, $A$ is called hermitian if $A=A^{*}$, unitary if $A A^{*}=E_{n}$, and normal if $A A^{*}=A^{*} A$. Clearly, any hermitian or unitary matrix is normal.

Lemma 2.3. Let $K$ be a formally real pythagorean field and let $A$ be a normal $n \times n$ matrix over $K(i), n \geq 2$, with characteristic polynomial $\chi_{A}$ such that $\chi_{A}=f \cdot g$ where $f, g \in K(i)[x]$ are nonconstant polynomials. If $f(x)=x-a$ or $f, g$ are relatively prime, then there exists a unitary $n \times n$ matrix $U$ over $K(i)$ such that

$$
U^{*} A U=\left(\begin{array}{cc}
B & 0 \\
0 & B^{\prime}
\end{array}\right),
$$


where $B$, resp. $B^{\prime}$, is a normal $m \times m$, resp. $m^{\prime} \times m^{\prime}$, matrix over $K(i)$ such that $m, m^{\prime} \geq 1$.

Proof. Put $F=K(i)$ and let $\varphi: F^{n} \rightarrow F^{n}$, resp. $\varphi^{*}: F^{n} \rightarrow F^{n}$, be the linear mapping defined by $\varphi(v)=A v$, resp. $\varphi^{*}(v)=A^{*} v$. We show that there exists a proper subspace $V \neq 0$ of $F^{n}$ such that $\varphi(V) \subseteq V$ as well as $\varphi^{*}(V) \subseteq V$.

If $a \in F$ is an eigenvalue of $A$ with corresponding eigenvector $v$, then

$$
\begin{aligned}
0 & =\langle\varphi(v)-a v, \varphi(v)-a v\rangle \\
& =\langle\varphi(v), \varphi(v)\rangle-\langle\varphi(v), a v\rangle-\langle a v, \varphi(v)\rangle+\langle a v, a v\rangle \\
& =\left\langle\varphi^{*}(v), \varphi^{*}(v)\right\rangle-\left\langle a^{*} v, \varphi^{*}(v)\right\rangle-\left\langle\varphi^{*}(v), a^{*} v\right\rangle+\left\langle a^{*} v, a^{*} v\right\rangle \\
& =\left\langle\varphi^{*}(v)-a^{*} v, \varphi^{*}(v)-a^{*} v\right\rangle,
\end{aligned}
$$

i.e. $\varphi^{*}(v)=a^{*} v$ and we put $V=[v]$. If $\chi_{A}(a) \neq 0$ for all $a \in F$, then $\chi_{A}$ has at least two distinct irreducible divisors by assumption. As in the proof of Lemma 2.1 there exists a proper subspace $W \neq 0$ of $F^{n}$ such that $\varphi(W) \subseteq W$. Let $\operatorname{dim} W$ be minimal and let $\chi$ be the characteristic polynomial of $\left.\varphi\right|_{W}$. Again, as in the proof of Lemma 2.1 there exists a proper subspace $U \neq 0$ of $W$ such that $\varphi(U) \subseteq U$ whenever $\chi$ is reducible, which would contradict the minimality of $\operatorname{dim} W$. Thus $\chi$ is irreducible and for any $k \in \mathbb{N}$ we define

$$
U_{k}=W+\varphi^{*}(W)+\cdots+\varphi^{* k}(W) .
$$

Since $\varphi\left(\varphi^{* k}(W)\right)=\varphi^{* k}(\varphi(W)) \subseteq \varphi^{* k}(W)$, all of the $\varphi^{* k}(W)$ as well as $U_{k}$ are $\varphi$-invariant. By the minimality of $\operatorname{dim} W \geq \operatorname{dim} \varphi^{* k}(W)$ we conclude

$$
U_{k-1} \cap \varphi^{* k}(W)=0 \text { or } \varphi^{* k}(W) \subseteq U_{k-1} .
$$

This means that there exists $k \in \mathbb{N}$ such that

$$
U_{k}=W \oplus \varphi^{*}(W) \oplus \cdots \oplus \varphi^{* k}(W), \varphi^{* k}(W) \neq 0 \text { and } \varphi^{* k+1}(W) \subseteq U_{k},
$$

i.e. $\varphi^{*}\left(U_{k}\right) \subseteq U_{k}$, and $U_{k}$ is a nonzero $\varphi$ - as well as $\varphi^{*}$-invariant subspace of $F^{n}$. We put $V=U_{k}$, and to show that $V$ is proper we assume $U_{k}=F^{n}$. Then $\chi_{A}=\chi_{\varphi}=\chi^{k+1}$, which is a contradiction since $\chi_{A}$ has at least two different irreducible divisors.

We turn to the proof of Lemma 2.3. Letting $V$ be as above we choose an orthonormal basis $\left\{v_{1}, \ldots, v_{l}\right\}$ of $V$ which can be completed to an orthonormal basis $\left\{v_{1}, \ldots, v_{n}\right\}$ of $F^{n}$. Again, let $U$ be the $n \times n$ matrix over $F$ whose $i$-th column vector equals $v_{i}$. Clearly, $U$ is unitary and $\left\langle v_{i}, A v_{j}\right\rangle=0$ for $i>l$ and $j \leq l$ since $V$ is $\varphi$-invariant and $\left\langle v_{i}, A v_{j}\right\rangle=\left\langle A^{*} v_{i}, v_{j}\right\rangle=0$ for $i \leq l, j>l$ since $V$ is $\varphi^{*}$-invariant.

Corollary 2.4. Let $K$ be formally real pythagorean and let $A$ be a normal matrix over $K(i)$ such that the characteristic polynomial splits completely over $K(i)$. Then there exists a unitary matrix $U$ over $K(i)$ such that $U^{*} A U$ is diagonal.

If $K$ is real closed, then $K(i)$ is algebraically closed and $\chi_{A}$ splits completely into linear factors over $K(i)$. By Corollary 2.4 there exists a unitary matrix $U$ over $K(i)$ such that $U^{*} A U$ is diagonal where the diagonal elements $a_{l}+i b_{l}\left(a_{l}, b_{l} \in K\right)$ are precisely the eigenvalues of $A$. More generally, if $K$ is an intersection of real closed fields $K_{\nu}$, then $K$ is formally real pythagorean. Since $2 a_{1}, \ldots, 2 a_{n}$ are the eigenvalues of $A^{*}+A$ and $2 b_{1}, \ldots, 2 b_{n}$ the eigenvalues of $i\left(A^{*}-A\right)$, we conclude $a_{1}, \ldots, a_{n}, b_{1}, \ldots, b_{n} \in K$, i.e., $\chi_{A}$ splits completely over $K(i)$, and this shows 
Corollary 2.5. Let $K$ be an intersection of real closed fields and let $A$ be a normal $n \times n$ matrix over $K(i)$. Then there exists a unitary matrix $U$ over $K(i)$ such that $U^{*} A U$ is diagonal.

Valuation theory. Here we give some basic definitions from valuation theory which are important in connection with complete fields and Hensel's Lemma as well as formal power series.

Let $K$ be a field; by a valuation on $K$ we understand a real-valued function $v: K \rightarrow \mathbb{R}_{0}^{+}$such that the following hold for all $x, y \in K$ :

(V1) $v(x)=0$ if and only if $x=0$.

(V2) $v(x+y) \leq \max \{v(x), v(y)\}$.

(V3) $v(x y)=v(x) v(y)$.

Given any valuation $v$ on $K$, we define

$$
B_{v}=\{x \in K \mid v(x) \leq 1\} .
$$

It is easily verified that $B_{v}$ is a subring of $K$ which is called the valuation ring of $v$ and moreover

$$
M_{v}=\{x \in K \mid v(x)<1\}
$$

is the unique maximal ideal of $B_{v}$, i.e., $B_{v}$ is a local ring. The residue class ring $B_{v} / M_{v}$ is a field which is called the residue class field of $v$ denoted by $\overline{B_{v}}$ and the canonical homomorphism

$$
-: B_{v} \longrightarrow \overline{B_{v}}, b \longmapsto \bar{b}
$$

can be extended to a homomorphism of the corresponding polynomial ring via

$$
\begin{gathered}
-: B_{v}[x] \longrightarrow \overline{B_{v}}[x], \\
a_{n} x^{n}+a_{n-1} x^{n-1}+\ldots+a_{0} \longmapsto \overline{a_{n}} x^{n}+\overline{a_{n-1}} x^{n-1}+\ldots+\overline{a_{0}} .
\end{gathered}
$$

The following example is central in the application of valuation theory in geometry and complex analysis:

Let $k$ be any field and let $k(x)$ be the rational function field of $k$ in one variable $x$. We choose a polynomial $p$ over $k$ which is irreducible over $k$. Then any nonzero rational function $q$ in $x$ over $k$ can be written as

$$
q=p^{\nu} \cdot \frac{f}{g}, \quad \nu \in \mathbb{Z} ; f, g \in k[x] ; p \nmid f g .
$$

We define $v_{p}$ by setting

$$
v_{p}(q)=\delta^{\nu}
$$

where $\delta \in \mathbb{R}, 0<\delta<1$ is arbitrary but fixed and $v_{p}(0)=0$. Then $v_{p}$ is a valuation on $k(x)$ which is called the $p$-adic valuation on $k(x)$. If $k$ is algebraically closed, then $p$ is linear and we may take $p(x)=x-a$ for some $a \in k$. In this case $B_{v}$ consists of all rational functions for which $a$ is not a pole. If $a$ is a pole or a zero of $q$, then $\nu$ is the corresponding order.

Any field $K$ with valuation $v$ possesses a completion $\hat{K}$ which is characterized by the following properties.

(C1) $\hat{K}$ is a field extension of $K$ with valuation $\hat{v}$ which extends $v$, i.e., $v(x)=$ $\hat{v}(x)$ for all $x \in K$.

(C2) $K$ is dense in $\hat{K}$, i.e., for any $\epsilon \in \mathbb{R}^{+}$and $x \in \hat{K}$ there exists $y \in K$, such that $\hat{v}(x-y)<\epsilon$.

(C3) $\hat{K}$ is complete, i.e., every Cauchy sequence in $\hat{K}$ is convergent in $\hat{K}$. 
In connection with complete fields the notation of henselian valuations is central. Here a valuation $v$ on $K$ is said to be henselian if the following statement is satisfied:

Hensel's Lemma (HL1). Let $F \in B_{v}[x]$ be a monic polynomial. If there exist monic polynomials $g, h \in \overline{B_{v}}[x]$ such that $\bar{F}=g \cdot h$ and $g, h$ are relatively prime in $\overline{B_{v}}[x]$, then there exist monic polynomials $G, H \in B_{v}[x]$ such that $\bar{G}=g, \bar{H}=h$, and $G \cdot H=F$.

Henselian valuations and Hensel's Lemma have many applications in various branches of mathematics, e.g., algebraic number theory, algebraic geometry, and the theory of finite-dimensional division algebras. The classical example of henselian valuations is given by complete valued fields, i.e., if $v$ is a valuation on $K$ such that $K$ is complete with respect to $v$, then $v$ is henselian. But in general, henselian valued fields need not be complete. In order to prove that a given valuation is henselian it is very useful to have equivalent versions of Hensel's Lemma which are simpler to verify than the one given above (cf. $[\mathrm{E}, \mathrm{R}])$.

Hensel's Lemma (HL2). Let $f \in B_{v}[x]$ be a monic polynomial. If there exists $a \in B_{v}$ such that $\bar{a}$ is a simple root of $\bar{f}$ in $\overline{B_{v}}$, then there exists $A \in B_{v}$ such that $f(A)=0$ and $\bar{A}=a$.

Clearly, in the version above we can restrict to the case where $\bar{a}=\overline{0}$ since $f(x)=f(x-a+a)=g(x-a)$ where $g(x) \in B_{v}[x]$ is monic such that $\overline{0}$ is a simple root of $\bar{g}$ in $\overline{B_{v}}$.

We give an example. Let $k$ be a field, $a \in k$, and let $v_{x-a}$ be the $(x-a)$-adic valuation on $k(x)$. Then the completion of $k(x)$ with respect to $v_{x-a}$ is the field $k((x-a))$ of all formal Laurent series in $x-a$ (cf. [E, Section 5]), where the nonzero elements of $k((x-a))$ can be expressed in the form

$$
\varphi=\sum_{i=\nu}^{\infty} a_{i}(x-a)^{i}=a_{\nu}(x-a)^{\nu}+a_{\nu+1}(x-a)^{\nu+1}+\ldots,
$$

where $\nu \in \mathbb{Z}$ and $a_{\nu} \neq 0$. The sum $\psi+\varphi$ of two Laurent series $\psi$ and $\varphi$ is defined by adding the coefficients of the same $(x-a)$-powers and the product $\psi \cdot \varphi$ is the usual Cauchy product. With respect to these operations $k((x-a))$ is indeed a field and $\hat{v}_{x-a}$ is defined by $\hat{v}_{x-a}(0)=0$ and

$$
\hat{v}_{x-a}(\varphi)=\hat{v}_{x-a}\left((x-a)^{\nu}\right)=\delta^{\nu},
$$

where $\varphi$ and $\delta$ are as above. Furthermore,

$$
\begin{aligned}
& B_{\hat{v}_{x-a}}=\left\{a_{0}+a_{1}(x-a)+a_{2}(x-a)^{2}+\ldots \mid a_{0}, a_{1}, a_{2}, \ldots \in k\right\}, \\
& M_{\hat{v}_{x-a}}=\left\{\quad a_{1}(x-a)+a_{2}(x-a)^{2}+\ldots \mid a_{0}, a_{1}, a_{2}, \ldots \in k\right\}
\end{aligned}
$$

and $\overline{B_{\hat{v}_{x-a}}}=k$. Since $k((x-a))$ is complete with respect to $\hat{v}_{x-a}$ we conclude that $\hat{v}_{x-a}$ is henselian.

The Principal Axis Theorem. A field $K$ is said to have the Principal Axis Property if for any symmetric matrix $A$ over $K$ there exists an orthogonal matrix $U$ over $K$ such that $U^{t} A U$ is diagonal. For instance, $K$ has the Principal Axis Property if $K=\mathbb{R}$ or more generally if $K$ is real closed. By Corollary 2.2, $K$ has also the Principal Axis Property if $K$ is an intersection of real closed fields.

Theorem 2.6. Let $K$ be a field with henselian valuation $v$. If $\overline{B_{v}} \cong \mathbb{R}$, then $K$ is formally real pythagorean. Furthermore, $K$ has the Principal Axis Property. 
Proof. First of all we show that $K$ is formally real pythagorean. Let $a_{1}, \ldots, a_{n}$ be nonzero elements of $K$ such that $a_{1}^{2}+\ldots+a_{n}^{2}=0$ and let $v\left(a_{1}\right) \geq v\left(a_{i}\right), i \geq 2$. We can assume that $v\left(a_{1}\right)=1$, since otherwise we divide both sides of the equation by $a_{1}$. After passing to the residue class field we obtain ${\overline{a_{1}}}^{2}+\ldots+{\overline{a_{n}}}^{2}=0$, which is a contradiction since $\overline{a_{1}}=\overline{1}$ and $\overline{B_{v}} \cong \mathbb{R}$ is formally real. To show that $K$ is pythagorean let $a, b$ be nonzero elements of $K, v(a) \geq v(b)$. Then $v\left(a^{-1} b\right) \leq 1$ and $\overline{1}+{\overline{a^{-1} b}}^{2} \neq 0$. Thus, $x^{2}=\overline{1}+{\overline{a^{-1}}}^{2}$ has two distinct roots which shows that $x^{2}=1+\left(a^{-1} b\right)^{2}$ has a solution $c$ in $K$ by Hensel's Lemma and $a^{2}+b^{2}=(a c)^{2}$.

We turn to the main part of the proof. Let $A=\left(a_{i j}\right)$ be a symmetric $n \times n$ matrix over $K$ and we prove the theorem by induction on $n$ where the case $n=1$ is trivial. Since the claim holds true for $A$ if and only if it holds for $A-a_{11} E_{n}$, we are also assuming that $a_{11}$ is 0 . Furthermore, let $v\left(a_{\nu \mu}\right)=\max \left\{v\left(a_{i j}\right) \mid 1 \leq i, j \leq n\right\}>0$. Since the claim holds true for $A$ if and only if it holds for $a_{\nu \mu}^{-1} A$, we can finally assume that all entries of $A$ are in $B_{v}$ and that at least one entry is equal to 1 . Consider the $n \times n$ matrix $\bar{A}$ which can be obtained from $A$ by replacing each $a_{i j}$ by $\overline{a_{i j}}$. The field $\overline{B_{v}} \cong \mathbb{R}$ has the Principal Axis Property and therefore $\chi_{\bar{A}}=\overline{\chi_{A}}$ splits completely over $\overline{B_{v}}$ into linear factors. If at least two linear factors are different, then $\chi_{A}$ is reducible by Hensel's Lemma and we are done by the induction hypothesis and Lemma 2.1. It remains to discuss the case $\chi_{\bar{A}}= \pm(x-\bar{a})^{n}$ and we will show that this will lead to a contradiction. There exists an $n \times n$ matrix $U$ over $B_{v}$ such that $\bar{U}^{t} \bar{A} \bar{U}=\bar{a} E_{n}$, i.e. $\bar{A}=\bar{a} E_{n}$. By $\overline{a_{11}}=\overline{0}$ we conclude $\bar{a}=\overline{0}$ but we also know that at least one entry of $\bar{A}$ must be equal to $\overline{1}$.

Remarks. 1) If $K$ is as in the theorem above, then the arguments used in the beginning of the proof of Theorem 2.6 show that $a_{1}, \ldots, a_{n} \in B_{v}$ for all $a_{1}, \ldots, a_{n} \in$ $K$ whenever $a_{1}^{2}+\ldots+a_{n}^{2} \in B_{v}$. Thus, if $U=\left(u_{i j}\right)$ is any orthogonal $n \times n$ matrix over $K$, then all $u_{i j}$ must be in $B_{v}$ and at least one $u_{i j}$ must be a unit in $B_{v}$ since $u_{i 1}^{2}+\ldots+u_{i n}^{2}=1$ for every $1 \leq i \leq n$. This shows that the entries of an orthogonal matrix $U$ lie in $B_{v}$ and that $\bar{U}$ is an orthogonal matrix over $\overline{B_{v}}$.

2 ) Since the proof above also shows that $K$ has the Principal Axis Property if $\overline{B_{v}}$ is real closed or an intersection of real closed fields, we obtain the following more general version:

Theorem 2.7. Let $K$ be a field with henselian valuation $v$. If $\overline{B_{v}}$ is an intersection of real closed fields, then $K$ has the Principal Axis Property.

Let $K$ be a field with valuation $v$ such that $\overline{B_{v}} \cong \mathbb{R}$ and we consider normal matrices over $K(i)$. Since $x^{2}+1$ is irreducible over $\overline{B_{v}}$, the valuation $v$ has a unique extension $v^{\prime}$ to $K(i)$ via

$$
v^{\prime}: K(i) \longrightarrow \mathbb{R}_{0}^{+}, a+i b \longmapsto \max \{v(a), v(b)\}
$$

and $\overline{B_{v^{\prime}}}=\overline{B_{v}}(i) \cong \mathbb{C}$. Therefore, $\left[\overline{B_{v^{\prime}}}: \overline{B_{v}}\right]=[\mathbb{C}: \mathbb{R}]=[K(i): K]$ and this means that $v$ is henselian if and only if $v^{\prime}$ is henselian. Under this assumption, an adaptation of the proof above (where Lemma 2.3 and Corollary 2.5 are used) shows immediately that normal matrices over $K(i)$ are diagonalizable by unitary matrices. Clearly, the same is true if $\overline{B_{v}}$ is real closed or an intersection of real closed fields (cf. Corollary 2.5) and this verifies

Theorem 2.8. Let $K$ be a field with valuation $v$ such that $\overline{B_{v}}$ is an intersection of real closed fields and let the unique extension $v^{\prime}$ of $v$ to $K(i)$ be henselian. Then 
for any normal matrix $A$ over $K(i)$ there exists a unitary matrix $U$ over $K(i)$ such that $U^{*} A U$ is diagonal.

Remarks. 1) In [CC, MSV] it is shown that the intersections of real closed fields are precisely the fields with the Principal Axis Property. Thus, in Theorems 2.7 and 2.8 the residue class field $\overline{B_{v}}$ can be any field with the Principal Axis Property.

2) The same arguments as used in Remark 1 after Theorem 2.6 show that the unitary matrix $U$ in Theorem 2.8 has entries in $B_{v^{\prime}}$.

3 ) The valuations considered in this paper are also called real valuations since the values are real numbers and only these valuations are interesting in our application to complex analysis. In a more general setting one usually deals with so-called Krull-valuations (cf. [E, Section 7]) and it is obvious to a reader who is familiar with this that Theorems 2.6, 2.7, and 2.8 also hold for Krull-valuations.

\section{The ANALYTIC PART}

We shall first discuss a local problem where the complex square matrix $A(z)=$ $\sum_{j=0}^{\infty}(z-a)^{j} A_{j}$ is a convergent power series in some complex neighborhood of $z=a$. Without loss of generality we shall always take $a=0$.

In the notation of the previous section, $K$ is the field of convergent Laurent series with real coefficients and $K(i)$ is the field of convergent Laurent series with complex coefficients (or alternatively the field of germs of meromorphic functions at $z=0$ ). A normal (hermitian) matrix over $K(i)$ thus corresponds to a meromorphic germ $A(z)$ which is normal (hermitian) for $z \in \mathbb{R}$. We remind the reader that the $*$ operation in $K(i)$ only acts on the coefficients in the series expansion of $A(z)$ and not on $z$. Thus, if $A(z)$ is considered as a function of $z \in \mathbb{C}$, normality as defined after Corollary 2.2 means normality for $z \in \mathbb{R}$ in the usual language of analysis. Let $v$, resp. $v^{\prime}$, be the $x$-adic valuation on $K$, resp. $K(i)$, i.e., if $f \in K$, resp. $f \in K(i)$, is a meromorphic germ at $z=0$, then $v(f)=v(x)^{n}$, resp. $v^{\prime}(f)=v^{\prime}(x)^{n}$, whenever $f$ has a zero or pole at $z=0$ of order $n$. Here we use the notation of Theorem 2.8. The valuation ring $B_{v}$, resp. $B_{v^{\prime}}=B_{v}(i)$, consists of all germs of holomorphic functions at $z=0$ (with real coefficients in the first case) and $\overline{B_{v}} \cong \mathbb{R}$, resp. $\overline{B_{v^{\prime}}} \cong \mathbb{C}$. Finally, $v^{\prime}$ is henselian since Hensel's Lemma in the form (HL2) holds true in view of the implicit function theorem for analytic functions [Hi]. Thus Theorem 2.8 and the subsequent Remark 2) imply Rellich's theorem:

Theorem 3.1. Near $z=0$, an analytic family of matrices $A(z)$ which is normal (hermitian) for $z \in \mathbb{R}$ can be diagonalized by an analytic family of matrices $U(z)$ which is unitary for real $z$. In particular, the eigenvalues, the associated eigenprojections and the associated eigenfunctions can be chosen to be germs of analytic functions.

We remark that the analogous theorem for formal power series in $z$ follows from the same arguments since the $x$-adic valuation $v^{\prime}$ on the field of formal Laurent series is henselian too as mentioned in Section 2. In applications to asymptotic perturbation theory (see e.g. [KS]) this result is often proven by hand, i.e. deduced from Theorem 3.1 by truncating the formal expansion of the matrix and checking that the expansions of the eigenvalues obtained for this polynomial approximations only differ in high orders in $z$ if the truncation is changed. Roughly speaking this extraction of the leading powers in $z$ is formalized in the algebraic machinery of the previous chapter. 
For the sake of completeness we shall next indicate how for the special case of analytic families of matrices the arguments of the previous section may be partly replaced by more pedestrian ones from analysis. The main problem arises from multiple eigenvalues of $A(0)$ which lead to a factorization

$$
\operatorname{det}(A(0)-t)=q(t)(t-b)^{k}
$$

of the characteristic polynomial of $A(0)$ for some $k>1$ and some polynomial $q(t)$ with $q(b) \neq 0$. Setting w.l.o.g. $b=0$, the Weierstrass preparation theorem (which replaces the implicit function theorem used in the case $k=1$ ) then provides a factorization

$$
\operatorname{det}(A(z)-t)=q(t, z)\left(t^{k}+a_{k-1}(z) t^{k-1}+\cdots+a_{0}(z)\right)=q(t, z) p(t, z)
$$

with coefficients analytic in $z$ such that $q(t, 0)=q(t)$. This factorization allows us to mimick the proof of Theorem 2.8 by use of (some form of) Lemma 2.3 without ever mentioning valuation theory.

In fact, the Weierstrass preparation theorem can thus be used to prove the stronger form (HL1) of Hensel's Lemma in this special case by applying it successively to each linear factor of the characteristic polynomial $\operatorname{det}(A(0)-t)$ (see $[\mathrm{F}])$.

Furthermore, even the use of the Weierstrass preparation theorem may be avoided by use of the Riesz projection and the standard calculus of residues. In the situation described by equation (1), let $\gamma$ be a contour encircling the multiple eigenvalue $b$ of $A(0)$ such that all the other eigenvalues of $A(0)$ are outside $\gamma$. Then, for $z$ near zero, the projection

$$
\Pi(z)=\frac{1}{2 \pi i} \int_{\gamma}(A(z)-t)^{-1} d t
$$

is well defined, has dimension $k$, and reduces $A(z)$. If $B(z)$ and $\hat{B}(z)$ denote the restriction of $A(z)$ to the range $\operatorname{Ran} \Pi(z)$ of $\Pi(z)$ and the complementary space $\operatorname{Ran} \hat{\Pi}(z)$, respectively, $\hat{\Pi}(z)=1-\Pi(z)$, one gets

$$
\operatorname{det}(A(z)-t)=\operatorname{det}(\hat{B}(z)-t) \operatorname{det}(B(z)-t),
$$

which proves the factorization in equation (2). Thus, using the Riesz projection $\Pi(z)$, one obtains a very simple version of the usual proof of the Weierstrass preparation theorem by the calculus of residues [H1]. Writing an arbitrary polynomial in $t$ as the characteristic polynomial of a matrix, it proves this theorem for polynomials in $t$.

Finally, we recall that the diagonalization of an analytic family $A(z)$, where $z$ belongs to a subdomain $\Omega \subset \mathbb{C}$ and $A(z)$ is normal on the real axis (intersected with $\Omega$ ), is of a more global nature than the local result in Theorem 3.1. Away from the real axis the eigenvalues, eigenprojections and (local choices of) eigenfunctions may have branchpoints or stronger algebraic singularities. However, on the real axis the eigenvalues, eigenprojections and even the eigenfunctions of $A(z)$ can be chosen to be analytic:

Theorem 3.2. Let $A(z)$ be an analytic family of matrices for $z \in \Omega \subset \mathbb{C}$ which is normal (hermitian) for $z \in \mathbb{R} \cap \Omega$. Then there is a complex neighborhood of $\mathbb{R} \cap \Omega$ on which $A(z)$ can be diagonalized by an analytic family of matrices $U(z)$ which is unitary for real $z$. In particular, the eigenvalues, the associated eigenprojections and the associated eigenfunctions can be chosen as germs of analytic functions on $\mathbb{R} \cap \Omega$. 
The analyticity of the eigenvalues and the eigenprojections follows from the local result in Theorem 3.1 because they are uniquely determined. The only subtle point concerns the possibility of choosing the eigenvectors (which then provide the diagonalizing matrix $U(z)$ ) globally as analytic functions of $z$. The standard reference for this fact is [Ka], where methods from the theory of differential equations are used to construct an analytic family of unitary matrices $U(z)$, globally for $z \in \mathbb{R}$, which diagonalizes $A(z)$. An alternative analytical approach is to use arguments from cohomology, i.e. basically the Mittag-Leffler theorem leading to "every holomorphic vector bundle over a noncompact Riemann surface is holomorphically trivial".

Here we shall provide a different proof which is purely algebraic: We show that Theorem 3.2 is an immediate consequence of Corollary 2.4 where a suitable field $K$ must be considered.

Algebraic proof. To apply the results of Section 2, we consider the matrix $A(z)$ as a square matrix with entries in the field $F$ of germs of meromorphic functions on $\mathbb{R} \cap \Omega$, i.e., each element $f \in F$ is represented by a function which is meromorphic in some neighborhood of $\mathbb{R} \cap \Omega$, and all functions which coincide in some neighborhood of $\mathbb{R} \cap \Omega$ are identified. $F$ is the quadratic extension $K(i)$ of the field $K$ of (germs of) real meromorphic functions on $\mathbb{R} \cap \Omega$. To apply Corollary 2.4 we shall show that $K$ is formally real pythagorean. Using the standard arguments of uniqueness of analytic continuation along $\mathbb{R} \cap \Omega$ it suffices to prove this locally for the field of real meromorphic germs at a point which without loss of generality can be taken as the origin. But this follows immediately from Theorem 2.6. Since the eigenvalues of $A(z)$ are analytic on $\mathbb{R} \cap \Omega$ the characteristic polynomial of $A(z)$ completely splits over $K(i)$ and thus $A(z)$ can be diagonalized by a unitary matrix $U(z)$ with entries in $K(i)$ in view of Corollary 2.4. Of course, the entries of $U(z)$ are actually analytic on $\mathbb{R} \cap \Omega$ since they are analytic in a neighborhood of each point in view of Remark 2) following Theorem 2.8 .

\section{REFERENCES}

[Ba] H. Baumgärtel, Endlichdimensionale analytische Störungstheorie, Akademie Verlag, 1972 MR 58:30389

[BK] E. Brieskorn, H. Knörrer, Ebene algebraische Kurven, Birkhäuser, 1981 MR 83i:14001

[C] P. M. Cohn, Puiseux's Theorem Revisited, J. Pure and App. Alg. 31 (1984), 1-4 MR 85d:14035; MR 89e:14022

[CC] A. Charnow, E. Charnow, Fields for which the principal axis theorem is valid, Math. Mag. 59 (1986), 222-225 MR 88c:12001a

[E] O. Endler, Valuation Theory, Springer, 1972 MR 50:9847

[F] G. Fischer, Ebene algebraische Kurven, Vieweg, 1994

[Hi] E. Hill, Analytic Function Theory Vol. I, Chelsea Publishing Company, 1976

[H1] L. Hörmander, The Analysis of Linear Partial Differential Operators I, Springer, 1990 MR 91m:35001b

[H2] L. Hörmander, An Introduction to Complex Analysis in Several Variables, North-Holland, 1966 MR 34:2933

[Ka] T. Kato, Perturbation Theory for Linear Operators, Springer, 1976 MR 53:11389

[KS] M. Klein, E. Schwarz, An elementary approach to formal WKB expansions in $\mathbb{R}^{n}$, Rev. Math. Phys. 2 (1990), 441-456 MR 92d:35213

[L] S. Lang, Algebra, Addison-Wesley, 1993

[MSV] D. Mornhinweg, D. B. Shapiro, K. G. Valente, The Principal Axis Theorem over Arbitrary Fields, Amer. Math. Monthly 100 (1993), 749-754 MR 94h:11020

[R] P. Ribenboim, Equivalent forms of Hensel's lemma, Expo. Math. 3 (1985), 3-24 MR 87a:12014 
[Re] F. Rellich, Störungstheorie der Spektralzerlegung, Math. Ann. 113 (1937), 600-619

[RS] M. Reed, B. Simon, Methods of Modern Mathematical Physics IV: Analysis of Operators, Academic Press, 1978 MR 58:12429c

[W] J. H. M. Wedderburn, On matrices whose coefficients are functions of a single variable, Trans. Amer. Math. Soc. 16 (1915), 328-332

Universität Potsdam, Institut für Mathematik, Postfach 601553, 14469 Potsdam, GERMANY

E-mail address: graeter@rz.uni-potsdam.de

E-mail address: mklein@math.uni-potsdam.de 